\title{
Transition-Metal-Free Direct C-H Arylation of Thiophene in Aqueous Media via Potassium Peroxymonosulfate
}

\author{
Sezer Özenler, ${ }^{[a]}$ Hakan Kaya ${ }^{[b]}$ Nuran Elmaci, ${ }^{[a]}$ and Umit Hakan Yildiz ${ }^{*[a]}$
}

This study covers the use of potassium peroxymonosulfate (PPS) which, is a mild and inexpensive catalyst, for direct coupling of $\mathrm{C}-\mathrm{H} / \mathrm{C}-\mathrm{H}$ for water soluble thiophene. The 3-(4methyl-3'- thienyloxy)propyltriethylammonium bromide (M1) has been selected as model monomer that reacted PPS in 2.0, 0.20 and 0.020 monomer to PPS ratio. The reaction has been monitored via NMR spectroscopy revealing the monomer consumption and formation of dimers to tetramers as well as completion of reaction within 15 minutes. Resultant product is easily collected without tedious work up steps. The computational calculation has optimized the regularity of the coupling

\section{Introduction}

Thiophene oligomers and polymers are often utilized as components of organic electronic devices; organic field effect transitors, ${ }^{[1-2]}$ photovoltaic, ${ }^{[3-4]}$ optoelectronic devices, ${ }^{[5]}$ light detectors, ${ }^{[6]}$ chemical and biological sensors ${ }^{[7-8]}$ due to their promising optical and electrical properties. The synthetic methodologies such as oxidative coupling ${ }^{[9-12]}$ and transition metal catalyzed aromatic carbon coupling ${ }^{[13-16]}$ are the most studied reactions to get gram scale products of oligo/ polythiophene and other polyconjugated molecules. These reactions are dependent transition metal catalyst that requiring halogenation of monomers as well as metal coordination that causing tedious purification steps and careful handling of monomers due to unstability. Recently, Direct arylation polymerization (DArP) ${ }^{[17-20]}$ is holding promises as a straightforward methodology on the other hand, $\mathrm{C}-\mathrm{H} / \mathrm{C}-\mathrm{H}$ coupling of thiophene by photoinitiator diphenyliodonium hexafluorophosphat ${ }^{[21]}$ is emerging as an alternative technique as compare to conventional aromatic coupling strategies. However most of these synthetic protocols need organic solvents which are mostly hazardous and expensive. Furthermore, trace amount of metal catalyst residues presents in resultant polymers exhibit toxic side effect for biological

[a] S. Özenler, Dr. N. Elmaci, Dr. U. H. Yildiz

Department of Chemistry, Izmir Institute of Technology, Izmir 35430 Turkey

E-mail: hakanyildiz@iyte.edu.tr

[b] H. Kaya

Department of Biotechnology and Bioengineering, Izmir Institute of Technology, Izmir 35430 Turkey

Supporting information for this article is available on the WWW under https://doi.org/10.1002/slct.201901508 as head to tail, tail to tail, head to tail (HT-TT-HT) for tetramer. The regularity of tetramer which is further satisfied via NMR analysis showing emergence of new peaks at 3.56 and 4.48 . The shifts in the UV maximum of reactive species have been monitored by absorbance spectroscopy showing time dependent red shift corresponds to growing from monomer to tetramers. The reaction has self-stopped due to low solubility of moderate condensates (higher than trimer), however further experiments have performed in water-organic mixtures showed great promises to obtain higher condensates.

application such as imaging and biosensing in physiological conditions.

Recent studies motivate transition metal free synthesis of conjugated polymer/oligomers in one pot. Maji et al. described transition-metal-free homocoupling of aryl, alkenyl, and alkynyl Grignard reagents by using the 2,2,6,6-tetramethyl-piperidine 1-oxyl radical (TEMPO) as oxidant. The described reaction has produced high yields for homocoupling reactions for synthesis of polyfluorenes, poly(fluorenylenebutadiynylenes), and poly (fluorenylenebutadienylenes). ${ }^{[22-25]}$ Another study presented by Cai et al. described a method to synthesize of $\pi$-conjugated polymers containing 1,3-butadiene units by polycondensation using an organic base as the catalyst instead of toxic transition metal-based catalysts. ${ }^{[26]}$ Their synthetic strategy was based on the reaction of $\alpha, \beta$-unsaturated compounds containing $\gamma-\mathrm{H}$ with aldehyde compounds, where electron-withdrawing groups such as ester and nitrile are incorporated as side groups. Although recent studies are holding promise for transition metal free synthesis in various organic solvents, however best of our knowledge transition metal free synthesis of conjugated oligomers/polymers in aqueous media is still unmet. The need for biocompatible synthetic protocols working in aqueous media are obvious. Here in this report we demonstrate the use of potassium peroxymonosulfate (PPS) which, is a mild and inexpensive catalyst, for direct coupling of $\mathrm{C}-\mathrm{H} / \mathrm{C}-\mathrm{H}$ in water soluble thiophene that eliminate complicated multistep reactions, and providing transition metal free oligomers. The new synthetic protocol for direct couplings of thiophene in aqueous media hold great promises to produce biocompatible thiophene oligomers. 


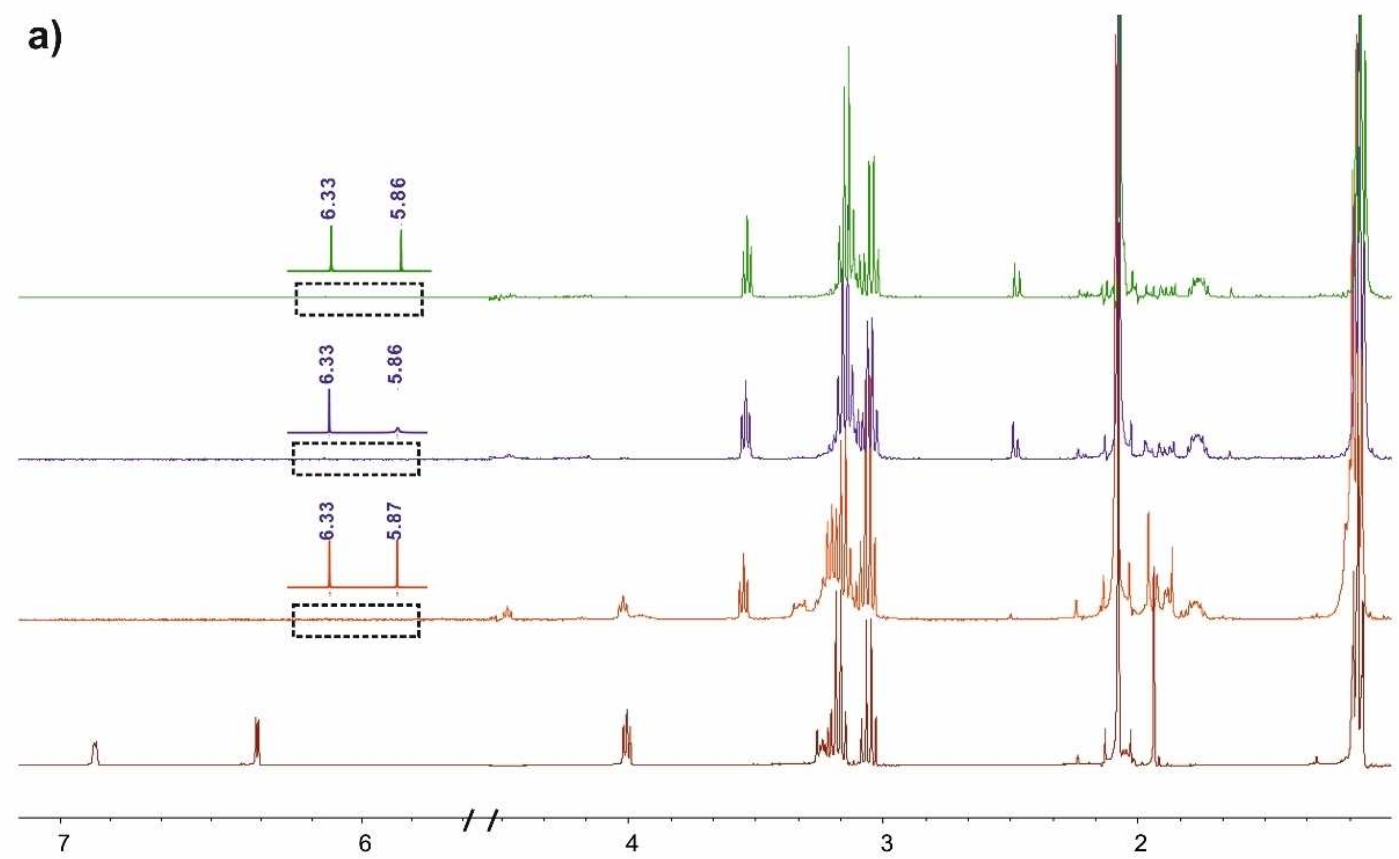

b)
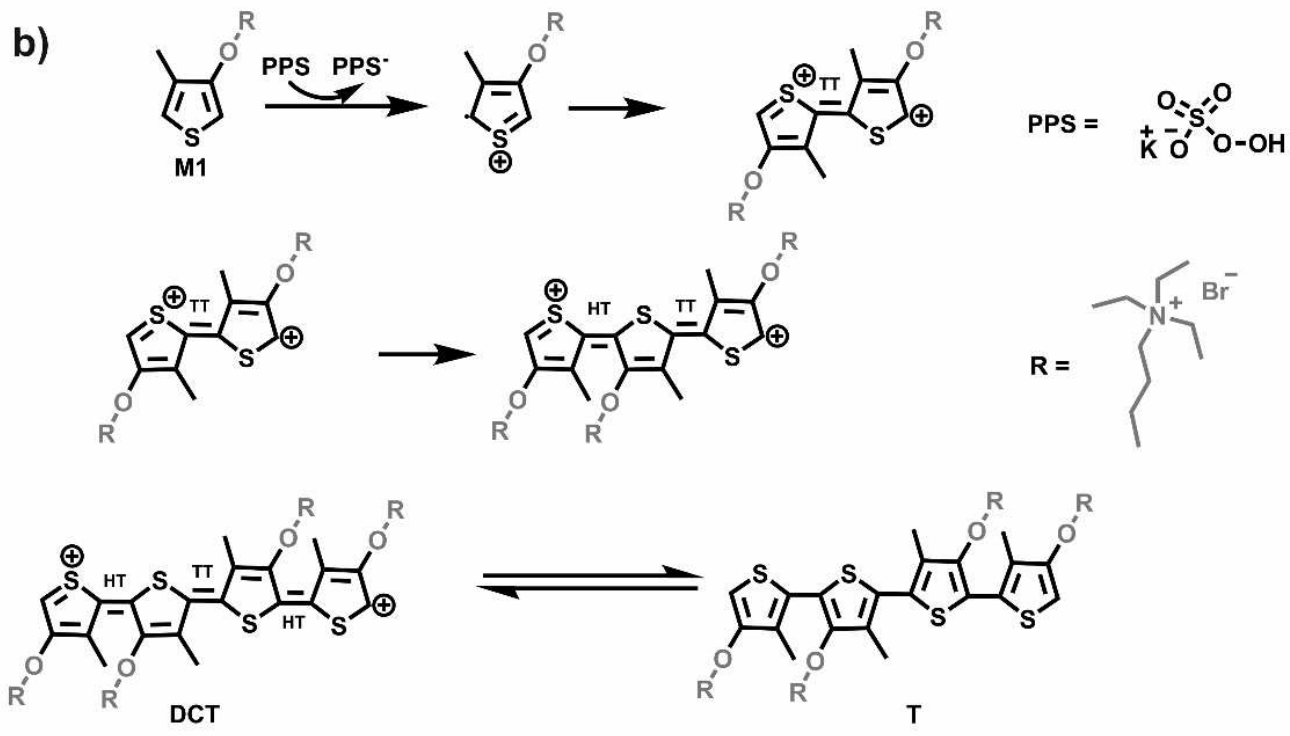

Figure 1. a) 1 HNMR spectrum of $M 1, M 1+P P S$ in 5 minutes, $M 1+P P S$ in 10 minutes and $M 1+P P S$ in 15 minutes, b) Mechanism of direct coupling of $C-H / C-H$ $(\mathrm{DCT}=$ dicationic tetramer, $\mathrm{T}=$ tetramer).

\section{Results and Discussion}

Coupling of thiophene by PPS was monitored by $1 \mathrm{H}-\mathrm{NMR}$ as shown in Figure 1a. The red spectra in stack plot is M1 $1 \mathrm{HNMR}$ (400 MHz, D2O, d): 6,89 (d, J=3 Hz, 1H, Ar H), 6.35 (d, J=3 Hz, $1 \mathrm{H}, \operatorname{Ar} \mathrm{H}) 4.02(\mathrm{t}, \mathrm{J}=6 \mathrm{~Hz}, 2 \mathrm{H} ; \mathrm{CH} 2), 3.24(\mathrm{~m}, \mathrm{~J}=5 \mathrm{~Hz}, 2 \mathrm{H} ; \mathrm{CH} 2)$ $3.12(\mathrm{~m}, \mathrm{~J}=7 \mathrm{~Hz}, 6 \mathrm{H}$; $(\mathrm{CH} 2) 3), 2.18(\mathrm{~s}, 3 \mathrm{H} ; \mathrm{CH} 3) 1.94(\mathrm{~m}, \mathrm{~J}=5 \mathrm{~Hz}$, $2 \mathrm{H} ; \mathrm{CH} 2), 1.13(\mathrm{~m}, \mathrm{~J}=7 \mathrm{~Hz}, 9 \mathrm{H} ;(\mathrm{CH} 3) 3)$. Prior to oxidizing reaction, 3-alkoxy-4-methylthiophene derivative the aromatic hydrogens at $2^{\prime}$ position and $5^{\prime}$ position yield a signal at 6.35 and $6.89 \mathrm{ppm}$ respectively. Upon addition of oxidizing agent
PPS, the intensity of aromatic hydrogens at 6.35 and $6.89 \mathrm{ppm}$ were significantly decreased due to positive charge localization on "S+" and free radical (the hydrogen neighboring to $\mathrm{S}+$ yields a small signal at $5.87 \mathrm{ppm}$ ). The intermediate radical species undergo a rapid coupling that produce dimers, trimers and predominantly tetramers (see Figure $1 \mathrm{~b}$ ). The most significant evolution in the spectrum has been observed at $4.02 \mathrm{ppm}$ $(t, 2 \mathrm{H}$ of $\mathrm{OCH} 2)$ that split into equally separated $(190 \mathrm{~Hz}$, or $0.46 \mathrm{ppm})$ three triplets and $4.48 \mathrm{ppm}(\mathrm{t}, \mathrm{J}=6 \mathrm{~Hz} 2 \mathrm{H})$. positioned at $3.56 \mathrm{ppm}(\mathrm{t}, \mathrm{J}=6 \mathrm{~Hz} 2 \mathrm{H}), 4.02 \mathrm{ppm}(\mathrm{t}, \mathrm{J}=6 \mathrm{~Hz} 2 \mathrm{H}$ 


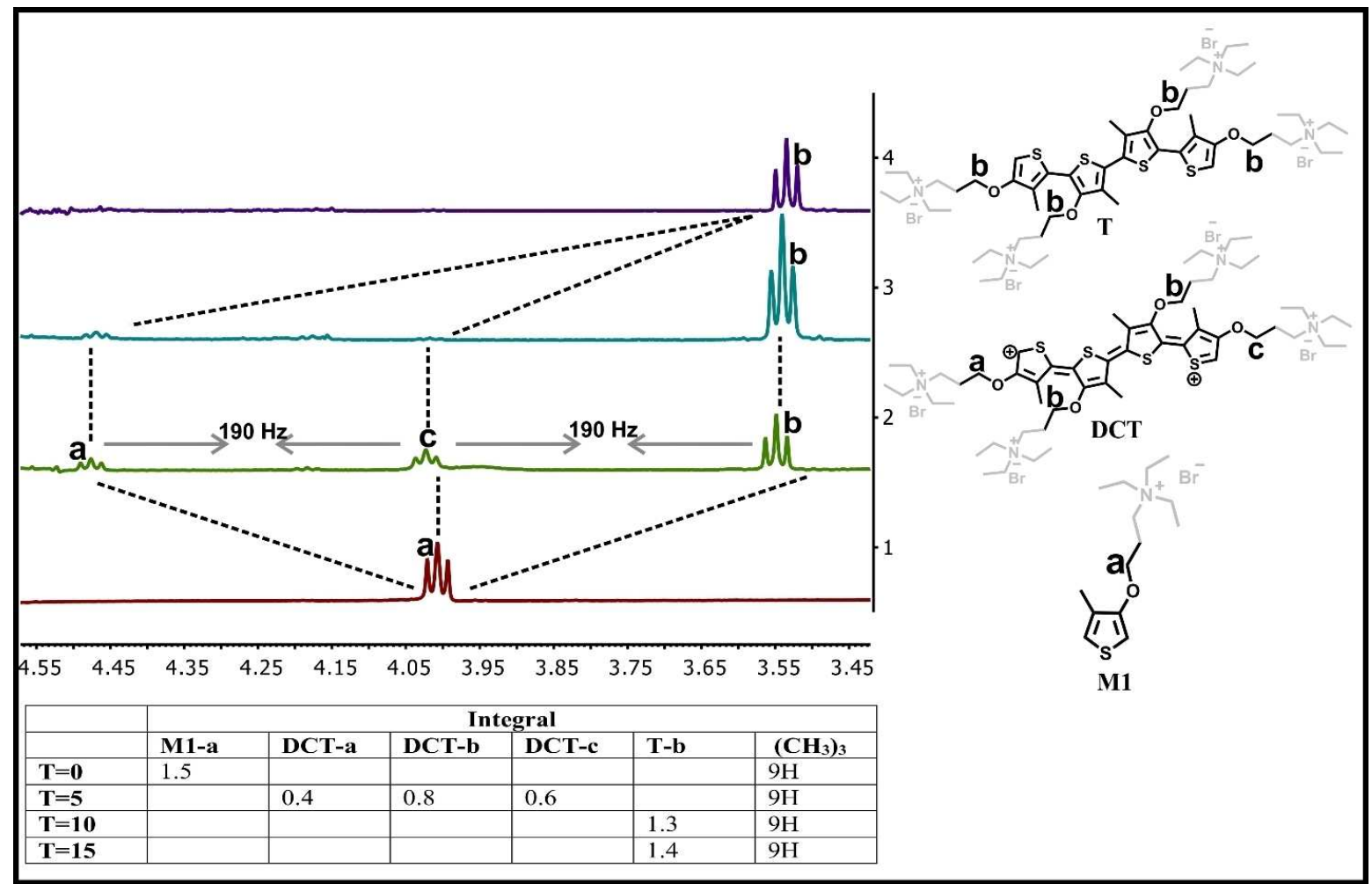

Figure 2. Magnified view of 1HNMR spectrum range between 3.45 to $4.55 \mathrm{ppm}$ band integral table of corresponding signal

The structure shown in Figure 2 (DCT) is the dication form of tetramer satisfy three triplets in above mentioned positions. Further integration analysis (see table in Figure 2) proves that central two thiophenes having an identical chemical environment position that $3.56 \mathrm{ppm}$ and terminal thiophenes positioned at $4.02 \mathrm{ppm}$ and $4.48 \mathrm{ppm}$ have 4:2:2 hydrogens respectively. Based on our computational calculations, regularity of the coupling is deduced as HT-TT-HT for tetramer which is satisfied by equal positioning of two central thiophenes $\mathrm{O}-\mathrm{CH} 2$ (4 hydrogens) and significant shift $(190 \mathrm{~Hz})$ of terminal thiophenes. The quinoid to aromatic transformation occurs by the completion of the reaction therefore three triplets positioned at 3.56, 4.02 and $4.48 \mathrm{ppm}$ merged together yielding single triplet at $3.56 \mathrm{ppm}$. (The NMR spectra of intermediate steps shown in supporting information section in S24, S25, S26, S27, S28.)

The relative energy for the coupling of two monomers were found to be; $0 \mathrm{kj} / \mathrm{mol}, 9,42 \mathrm{kj} / \mathrm{mol}$ and $17,62 \mathrm{kj} / \mathrm{mol}$ for $\Pi$, HT and $\mathrm{HH}$ respectively. Therefore, dimerization reaction is assumed to produce mostly $\Pi T$ since it is energetically favorable. $\pi$ dimer has two possibilities of formation of trimer either $\Pi \mathrm{T}-\mathrm{HT}(0 \mathrm{kj} / \mathrm{mol})$ or $\Pi \mathrm{T}-\mathrm{HH}(1,90 \mathrm{kj} / \mathrm{mol})$ and $\Pi \mathrm{T}-\mathrm{HT}$ trimer has four possibilities of formation of tetramer that HT-TT-HT was calculated as most stable structure (Figure 3).

In order to explore reaction character; UV measurement was applied repeatedly with respect to the time at constant temperature. M1 has two peaks which have absorbance maximum at $224 \mathrm{~nm}$ and $248 \mathrm{~nm}$. Addition of PPS to the

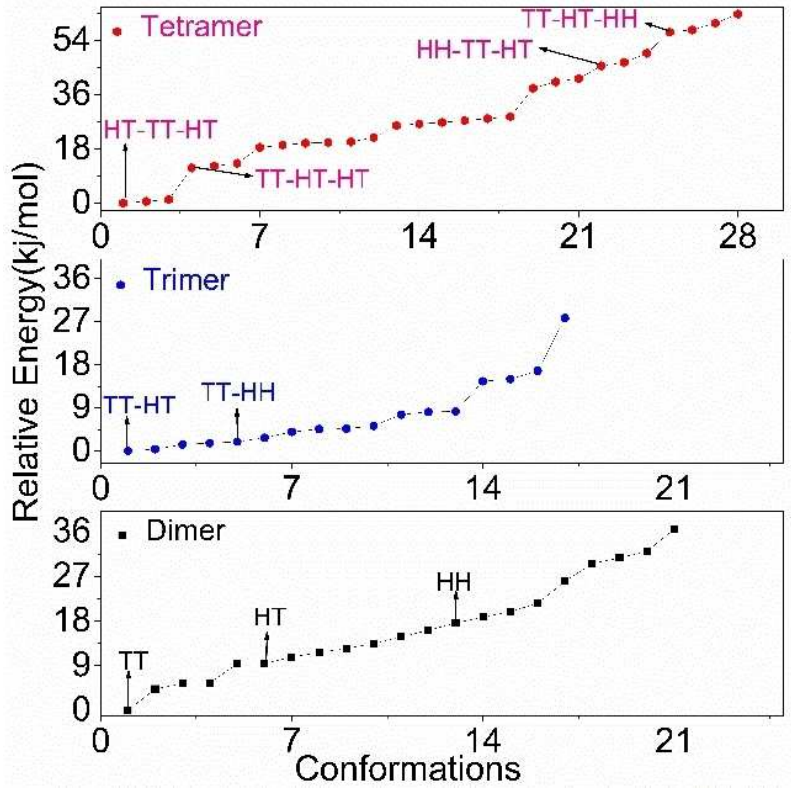

Figure 3. Relative energy graph of conformations of dimer, trimer and tetramer

monomer solution which is in distilled water, $224 \mathrm{~nm}$ and $248 \mathrm{~nm}$ peaks unchanged while a peak at nearly $704 \mathrm{~nm}$ was observed instantly in UV measurement (Figure 4a). This peak corresponds radical cation of thiophene monomer. ${ }^{[27-28]}$ The 

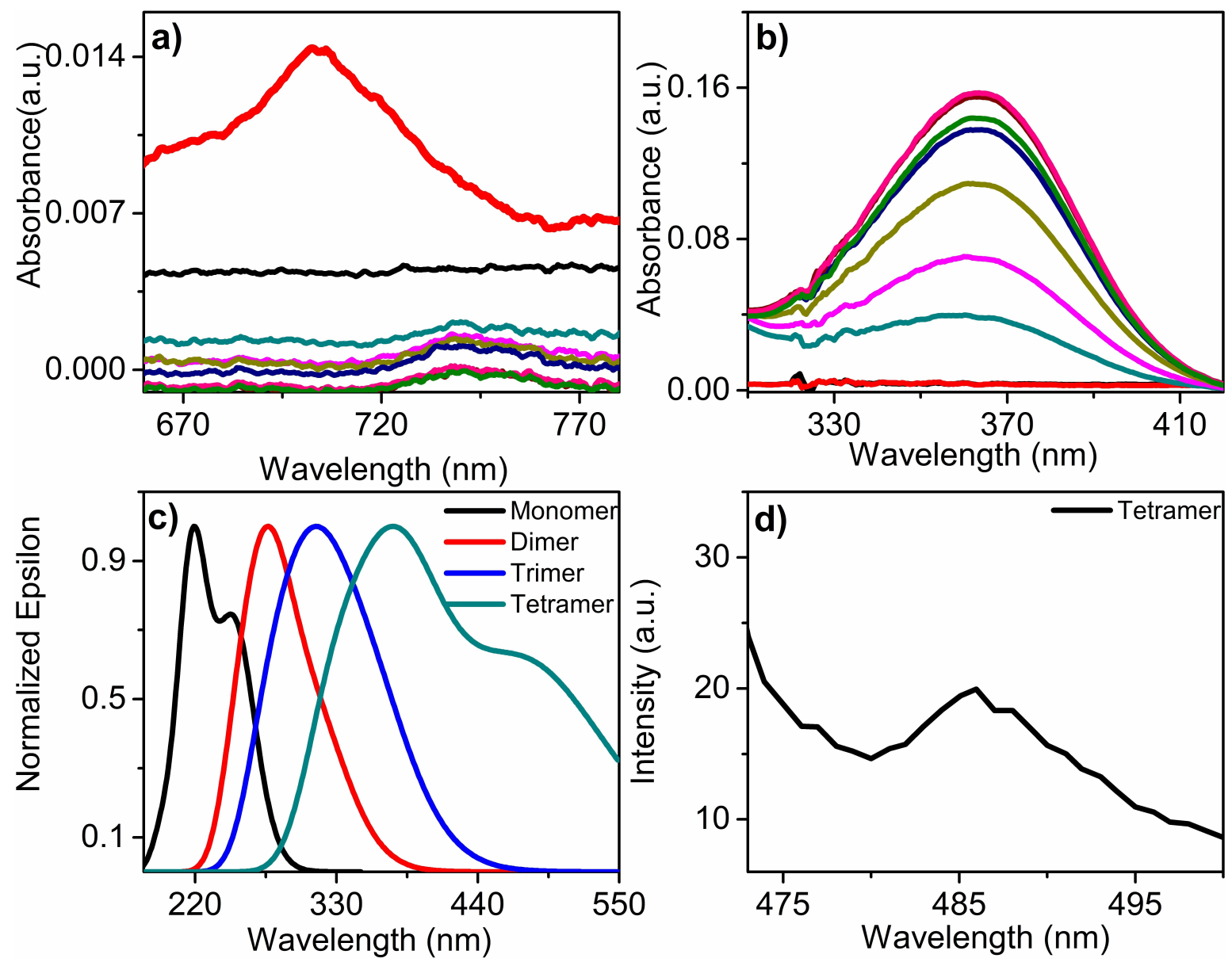

Figure 4. a, b) Absorbance spectrum of reaction upon heating of M1 + PPS in aqueous media over 20 minutes c) Theoretical spectra of oligothiophenes d) Fluorescence spectra of tetramer $K$ max excitation $=460 \mathrm{~nm}$ in DMF (black line: M1, red line: M1 + PPS, dark cyan: at 3 minutes, magenta line: at 6 minutes, dark yellow: at 9 minutes, navy line: at 12 minutes, green line: at 15 minutes, wine line: at 18 minutes and pink line: at 21 minutes for a and b).

two peaks that un-changed even after addition of PPS, are merged and obtain one peak at $240 \mathrm{~nm}$ during heating process. At the same time, in minute 6 , new peak is occurred at $360 \mathrm{~nm}$ and monomer peak at $240 \mathrm{~nm}$ is beginning to diminish (Figure 4b). We performed detailed spectrum analysis by deconvolution of Figure $4 \mathrm{~b}$. This analysis provides change in the distribution of trimer $(330 \mathrm{~nm})$ and tetramer $(360 \mathrm{~nm})$ individual peaks by reaction time (between 3 to 12 minutes). The intensity of trimer peak at $330 \mathrm{~nm}$ was found to be decreasing during the reaction period while tetramer peak $360 \mathrm{~nm}$ was intensifying. The intensity of the trimer peak at around 5th minutes was nearly tenfold lower than the tetramer peak assuring the depletion of trimer specie as compare the tetramer (see Figure S21 and S 22). By the detailed spectrum analysis, we concluded that nearly entire reaction tetramer is predominant specie and therefore NMR yielding tetramer characteristics rather than trimer or dimer. Newly occurred $360 \mathrm{~nm}$ peak increased with respect to the time however a peak gradually declines during further heating. Also, low intense peak occurs at nearly $738 \mathrm{~nm}$ which is responsible peak for quinodial quaternized thiophene (see Figure 4a). Occurrence of the $360 \mathrm{~nm}$ peak in virtue of oligomerization of the thiophene which level of the conjugation is increased, and red shift observed thanks to delocalization of the electron through their backbone. The identical procedure of coupling reaction has been provided without heating and the peak at $360 \mathrm{~nm}$ has not been observed therefore, we propose that the reaction is endothermic.

The theoretical UV-VIS spectra shown in Figure 4c, obtained by the most energetically favorable conformations of monomer, dimer, trimer and tetramer with absorbance maximum at $245 \mathrm{~nm}, 277 \mathrm{~nm}, 335 \mathrm{~nm}$ and $370 \mathrm{~nm}$ respectively. The absorbance maximum of all species is strongly dependent on both coupling type and dihedral angle among the monomers based on our calculation. Based on NMR results, HT-TT-HT coupling type selected as quaternized thiophene model for the computational works. The dihedral angles $(\theta 1, \theta 2, \theta 3)$ of the quaternized thiophene are $163^{\circ}-116^{\circ}-144^{\circ}$ and have $371 \mathrm{~nm}$ absorption maximum (see FIGURE 4c). Fluorescence spectra were also obtained for quaternized thiophene with excitation wavelength $460 \mathrm{~nm}$. The corresponding wavelength of maximum emission at $485 \mathrm{~nm}$ (see Figure 4d). Responsible peak is transition of electron through the zero level of the S1 to zero level of the S0. Low fluorescence intensity of short oligothio- 
phene chain is expected compared to long oligothiophene chain or polythiophene chain while M1 is fluorescently inactive.

Figure 5 shows the effect of initial PPS concentration on tetramer formation. Three different PPS concentrations

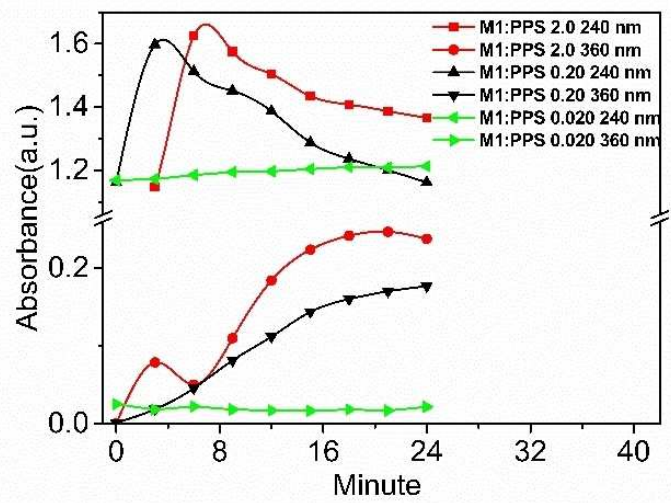

Figure 5. Change in the absorption maximum at 240 and $360 \mathrm{~nm}$ for 2.0, 0.20 and 0.020 monomer to PPS ratio.

$0,70 \mu \mathrm{mol}, 7,0 \mu \mathrm{mol}$ and $70,0 \mu \mathrm{mol}$ were used by keeping monomer (M1) concentration constant at 1,4 $\mu \mathrm{mol}$. (M1:PPS; $0.020,0.20$ and 2.0). There is no intensity increment at $360 \mathrm{~nm}$ for M1:PPS 0.020. While, M1:PPS increases to 0.20 and 2.0 significant absorbance change observed. The reaction rate has been calculated as $2,65 \mu \mathrm{g} / \mathrm{min}$ for M1:PPS 0.20 and 4,02 $\mu \mathrm{g} /$ min for M1:PPS 2.0. These results reveal that the oxidation of M1 is strongly dependent on initial concentration of PPS (as shown in figure $S 23$. UV-Vis measurements PPS added to M1 without heating shows no peak at $360 \mathrm{~nm}$ revealing temperature dependency of reaction.) The linear increase at the $360 \mathrm{~nm}$ tetramer peak observed for M1:PPS; 0.20 therefore we suggest that M1:PPS 0.2 is an optimal for proposed reaction.

Theoretical Raman spectra yield three major peaks at frequencies $1411 \mathrm{~cm}-1,1527 \mathrm{~cm}-1$ and $1596 \mathrm{~cm}-1$. referring to $\beta-\beta^{\prime}, \alpha-\alpha^{\prime}$, and mixing of $\alpha-\alpha^{\prime}, \alpha-\beta, \alpha^{\prime}-\beta^{\prime}$ stretching modes respectively shown in Figure $6 a$. In experimental Raman spectra (Figure $6 \mathrm{~b}$ ), there are three strong scattering which are $1391 \mathrm{~cm}-1,1532 \mathrm{~cm}-1$ and $1599 \mathrm{~cm}-1$. The experimental and computational frequencies of stretching mode are resembled to each other with a minor shift for instance $1596 \mathrm{~cm}-1$ was assumed to be identical to $1599 \mathrm{~cm}-1$, while $1527 \mathrm{~cm}-1$ identical to $1532 \mathrm{~cm}-1$ and $1391 \mathrm{~cm}-1$ identical to $1411 \mathrm{~cm}-1$. The peak at $1411 \mathrm{~cm}-1$ represent the $\beta-\beta^{\prime}$ stretching mode while at $1527 \mathrm{~cm}-1$ correspond dominantly $\alpha-\alpha^{\prime}$ and at $1596 \mathrm{~cm}-1$ represent the mixing of $\alpha-\alpha^{\prime}, \alpha-\beta$ and $\alpha^{\prime}-\beta^{\prime}$ stretching. In experimental Raman spectra the peak intensity at frequency $1532 / 1599 \mathrm{~cm}-1$ ratio is 0,85 while computational peak intensity at frequency $1527 / 1596 \mathrm{~cm}-1$ ratio is 3,58 . The significant difference between the intensity ratio two frequencies is due to the quinoid structure formation of the tetramer backbone. ${ }^{[29]}$ In view of these result, deconvolution of experimental spectra yield two peaks at $1599 \mathrm{~cm}-1$ and $1532 \mathrm{~cm}-1$, which are identical to theoretical spectra. The additional theoretical spectra shown in Figure $6 c$ proves that the emergence of two peaks at $1599 \mathrm{~cm}-1$ and $1532 \mathrm{~cm}-1$ as a result of tetramer formation. Therefore, the significant red shift in frequency incorporated with $\mathrm{C}=\mathrm{C}$ stretching mode is attributed to chain growth.
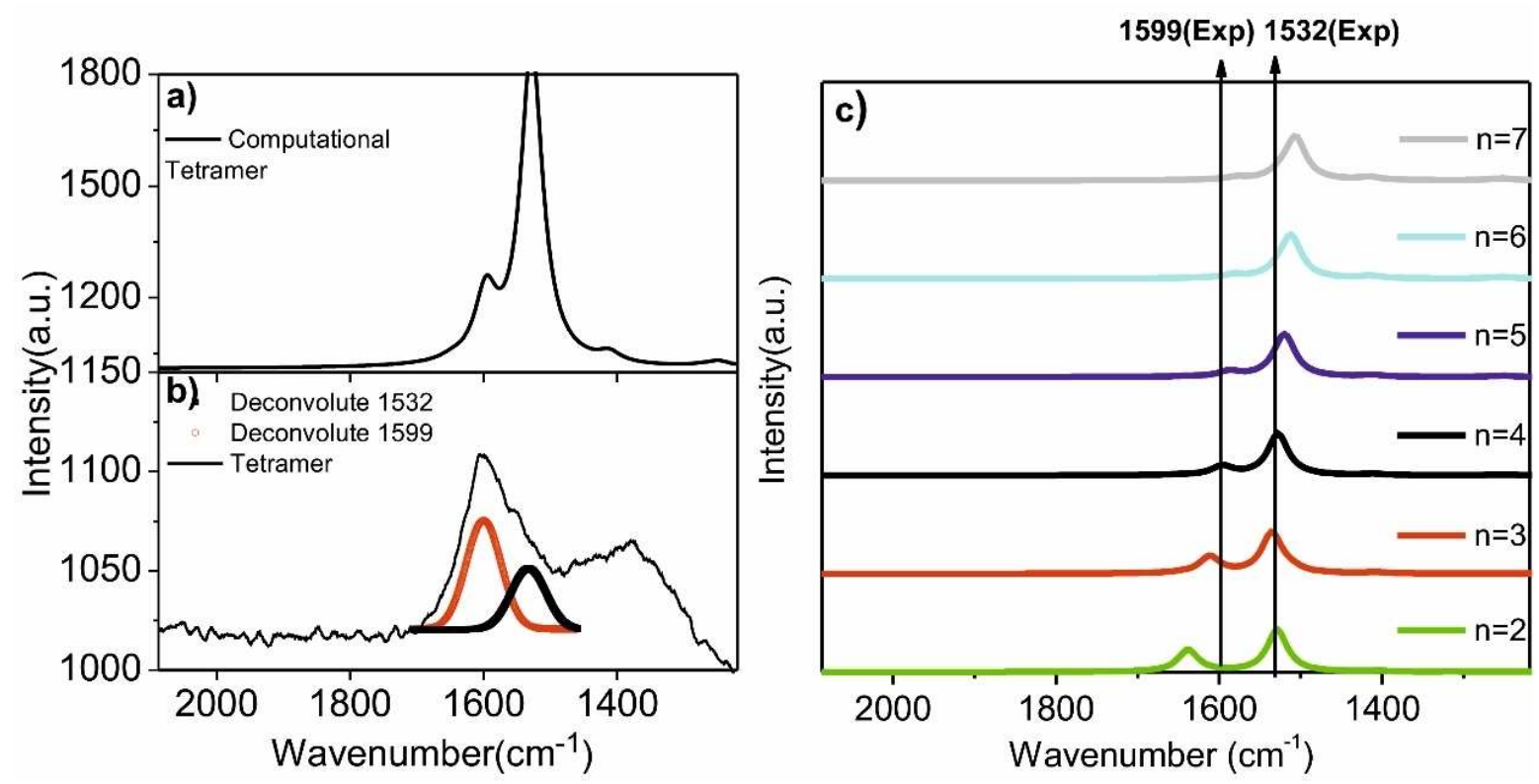

Figure 6. a) Computational tetramer Raman spectra b) experimental Raman spectra c) dimer to heptamer computational Raman spectra 


\section{Conclusions}

In this report, we have shown that direct coupling of $\mathrm{C}-\mathrm{H} / \mathrm{C}-\mathrm{H}$ in thiophene. Time dependent NMR and absorbance spectroscopy results proved that propagation occurs by oxidation of monomers. The regularity of conjugated condensates for instance tetramer were found to be HT-TT-HT. The reaction has self-stopped due to low solubility of moderate condensates (higher than trimer), however further experiments have performed in water-organic mixtures showed great promises to obtain higher condensates. The new synthetic protocol for direct couplings of thiophene in aqueous media hold great promises to produce biocompatible thiophene oligomers.

\section{Supporting information summary}

Experimental section, detailed of computation calculation method of substances, additional UV-Vis measurements results, NMR results provided in supporting information. Also, the movie which is the colour change of the reaction medium with respect to time at $50^{\circ} \mathrm{C}$ is available in the supporting information.

\section{Acknowledgements}

This work was supported by the The Scientific and Technological Research Council of Turkey (Project no: 116z547). The authors also thank to Dr. Huseyin Ozgener and Erhan Özdemir. Parts of this work (NMR) were carried out in, Izmir Institute of Technology (IZTECH). Author SÖ is YÖK 100-2000 scholarship holder.

\section{Conflict of Interest}

The authors declare no conflict of interest.

Keywords: Biocompatible oligothiophene $\cdot$ direct arylation in aqueous media $\cdot$ oligothiophene $\cdot$ transition metal free $\cdot$ watersoluble thiophene
[1] K. J. Baeg, M. Binda, D. Natali, M. Caironi, Y. Y. Noh, Adv. Mater. 2013, 25, 4267-4295.

[2] C. Dimitrakopoulos, P. R. L. Malenfant, Adv. Mater. 2002, 14, 99-117.

[3] D. Fichou, J. Mater. Chem. 2000, 10, 571-588.

[4] A. Facchetti, Chem. Mater. 2011, 23, 733-758.

[5] J. Janata, M. Josowicz, Nat. Mater. 2003, 2, 19-24.

[6] B. Kippelen, J.-L. Brédas, Energy Environ. Sci. 2009, 2.

[7] P. Lin, F. Yan, Adv. Mater. 2012, 24, 34-51.

[8] C. Wang, H. Dong, W. Hu, Y. Liu, D. Zhu, Chem. Rev. 2012, 112, 22082267.

[9] T.-A. Chen, X. Wu, R. D. Rieke, J. Am. Chem. Soc. 1994, 117, 233-244.

[10] S. Amou, O. Haba, K. Shirato, T. Hayakawa, M. Ueda, K. Takeuchi, M. Asai, J. Polym. Sci. A Polym. Chem. 1999, 37, 1943-1948.

[11] M. M. Bouman, E. E. Havinga, R. A. J. Janssen, E. W. Meijer, Mol. Cryst. Liq. Cryst. 2006, 256, 439-448.

[12] B. Carsten, F. He, H. J. Son, T. Xu, L. Yu, Chem. Rev. 2011, 111, 1493-1528.

[13] D. Iarossi, A. Mucci, L. Schenetti, R. Seeber, Macromolecules 1999, 32, 1390-1397.

[14] J. Pei, W.-L. Yu, W. Huang, A. J. Heeger, Macromolecules 2000, 33, 24622471.

[15] W. C. Wu, C. L. Liu, W. C. Chen, Polym. J. 2006, 47, 527-538.

[16] I. Osaka, R. D. Mccullough, Acc. Chem. Res. 2008, 41, 1202-1214.

[17] M. Lafrance, K. Fagnou, J. Am. Chem. Soc. 2006, 128, 16496-16497.

[18] B. t. Lie'gault, D. L. L. Caron, A. Vlassova, K. Fagnou, J. Org. Chem. 2008, 74, 1826-1834.

[19] Q. Wang, M. Wakioka, F. Ozawa, Macromol. Rapid Commun. 2012, 33, 1203-1207.

[20] J. A. Lee, C. K. Luscombe, ACS Macro Lett. 2018, 7, 767-771.

[21] Y. Yagci, F. Yilmaz, S. Kiralp, L. Toppare, Macromol. Chem. Phys. 2005, 206, 1178-1182.

[22] M. S. Maji, T. Pfeifer, A. Studer, Chem. - Eur. J. 2010, 16, 5872-5875.

[23] M. S. Maji, T. Pfeifer, A. Studer, Angew. Chem. 2008, 47, 9547-9550.

[24] A. Studer, M. Maji, Synthesis 2009, 2467-2470.

[25] A. Studer, T. Vogler, Synthesis 2008, 13, 1979-1993.

[26] X. Cai, Y. Liu, T. Lu, R. Yang, C. Luo, Q. Zhang, Y. Chai, Macromol. Rapid Commun. 2016, 37, 2005-2010.

[27] Y. Yagci, S. Jockusch, N. J. Turro, Macromolecules 2007, 40, 4481-4485.

[28] Y. Yagci, W. Schnabel, A. Wilpert, J. Bendig, J. Chem. Soc., Faraday Trans. 1994, 90, 287-291.

[29] J. Casado, V. Hernández, J. T. López Navarrete, Chem. Rec. 2015, 15, $1110-1118$

Submitted: April 26, 2019

Accepted: July 18, 2019 